\title{
Carpobrotus edulis (L.) N.E. Br. (Aizoaceae) y su presencia en la flora de Chile
}

\section{Carpobrotus edulis (L.) N.E. Br. (Aizoaceae) and its presence in the Chilean flora}

\author{
Gastón J. Sotes ${ }^{1,2 *}$, Lohengrin A. Cavieres ${ }^{1,2}$ \& Roberto Rodríguez ${ }^{1}$ \\ 1Departamento de Botánica, Facultad de Ciencias Naturales y Oceanográficas, Universidad de Concepción, Casilla \\ 160-C, Concepción, Chile. \\ ${ }^{2}$ Instituto de Ecología y Biodiversidad (IEB), Universidad de Chile, Casilla 653, Santiago, Chile. \\ *gastonsotes@gmail.com
}

\begin{abstract}
The genus Carpobrotus was represented on the Chilean flora only by native species $C$. chilensis. However, we found that the species native of South Africa $C$. edulis is naturalized in coastal areas. We report it for first time as an alien species for the Chilean territory.
\end{abstract}

La familia Aizoaceae está representada en Chile por 5 géneros (Carpobrotus, Galenia [introducido], Mesembryanthemum [introducido], Sesuvium [introducido] y Tetragonia) y unas 13 especies que habitan desde la Región de Arica y Parinacota a la Región de Los Lagos, encontrándose en suelos con baja disponibilidad hídrica como arenales y desiertos (Zuloaga et al. 2008).

Hasta la fecha, el género Carpobrotus sólo se encuentra representado en la flora de Chile por la especie nativa $C$. chilensis (Molina) N.E. Br. Esta especie ha sido descrita como frecuente en dunas litorales desde el norte (Región de Antofagasta) al sur del país (Región de Los Ríos) (Gay 1848, Reiche 1898, Muñoz Pizarro 1966, Zuloaga et al. 2008). Además, se ha reportado su presencia para Argentina, Australia, California, España y Nueva Zelanda (Munz 1959, Blake 1969, Castroviejo 1990, Zuloaga et al. 2008).

C. chilensis es una especie perenne y rastrera, hojas carnosas de $3-5 \mathrm{~cm}$ de largo, extendidas y levemente arqueadas, triangulares en corte transversal con las aristas totalmente lisas. Flores solitarias de $3-5 \mathrm{~cm}$ de diámetro con numerosos estaminodios petaloideos (pétalos) marginales de color purpúreo. Posee abundantes estambres y 10 estigmas, ambos de color amarillo (Muñoz Pizarro1966) (Fig. 1 A y C).

Sin embargo, al realizar un estudio sobre poblaciones litorales de Carpobrotus en las regiones de Valparaíso y del Biobío se localizó otra especie naturalizada que hasta ahora no había sido reportada como un componente de la flora chilena, identificada como Carpobrotus edulis (L.) N.E. Br. Esta especie es nativa de Sudáfrica y ha sido introducida en zonas templadas del mundo (Chile incluido) para jardinería y control de erosión de taludes en carreteras y zonas costeras (Schmalzer \& Hinkle 1987).

C. edulis es considerada una especie muy invasora en el litoral de Australia, Estados Unidos, Nueva Zelanda y sur de Europa (Global Invasive Species Database-sitio web). Se ha observado que en la Península Ibérica $C$. edulis invade zonas de dunas, donde modifica una serie de características del suelo, afectando negativamente el reclutamiento de especies nativas (Novoa et al. 2013, 2014). Sumado a esto, se ha reportado que $C$. edulis hibridiza con $C$. chilensis en California y con Aizoáceas originarias de Nueva Zelanda, lo cual genera una amenaza sobre la persistencia de las especies nativas (Albert et al. 1997, Heenan \& Sykes 2010). Junto con esto, aumenta el contenido de materia orgánica presente en las dunas costeras, promoviendo la invasión de otras especies invasoras (DiTomaso \& Healy 2007). Es de esperar que la confirmación de la presencia de esta importante especie invasora en Chile motive el estudio de su extensión geográfica y su impacto sobre las especies nativas.

\section{DESCRIPCIÓN DE LA ESPECIE}

Carpobrotus edulis (L.) N.E. Brown, The Genera of South African Flowering Plants 249 (1926).

Nombres vulgares: doca, uña de gato.

Hierba perenne, rastrera, suculenta, tallo hasta $2 \mathrm{~m}$ de longitud. Hojas carnosas de 7-10 cm de largo y de 1-1,5 $\mathrm{cm}$ de ancho, erectas y ligeramente curvadas, triangulares transversalmente con el ápice de la arista inferior suavemente aserrado (Fig. 1 D). Flores solitarias de $6-10 \mathrm{~cm}$ de diámetro con ovario ínfero. Perianto con el tubo turbinado, con 5 tépalos, los tres externos oblongos de 2-4,5 cm y los dos internos menores con el margen anchamente escarioso. Numerosos estaminodios petaloideos libres (pétalos), linear lanceolados, de color amarillento, rosáceo o purpúreo, con matices blancos. Estambres abundantes, primero erectos y luego convergentes hacia los estigmas, con los filamentos de color amarillo. Estigmas 10 en disposición radial, sésiles 

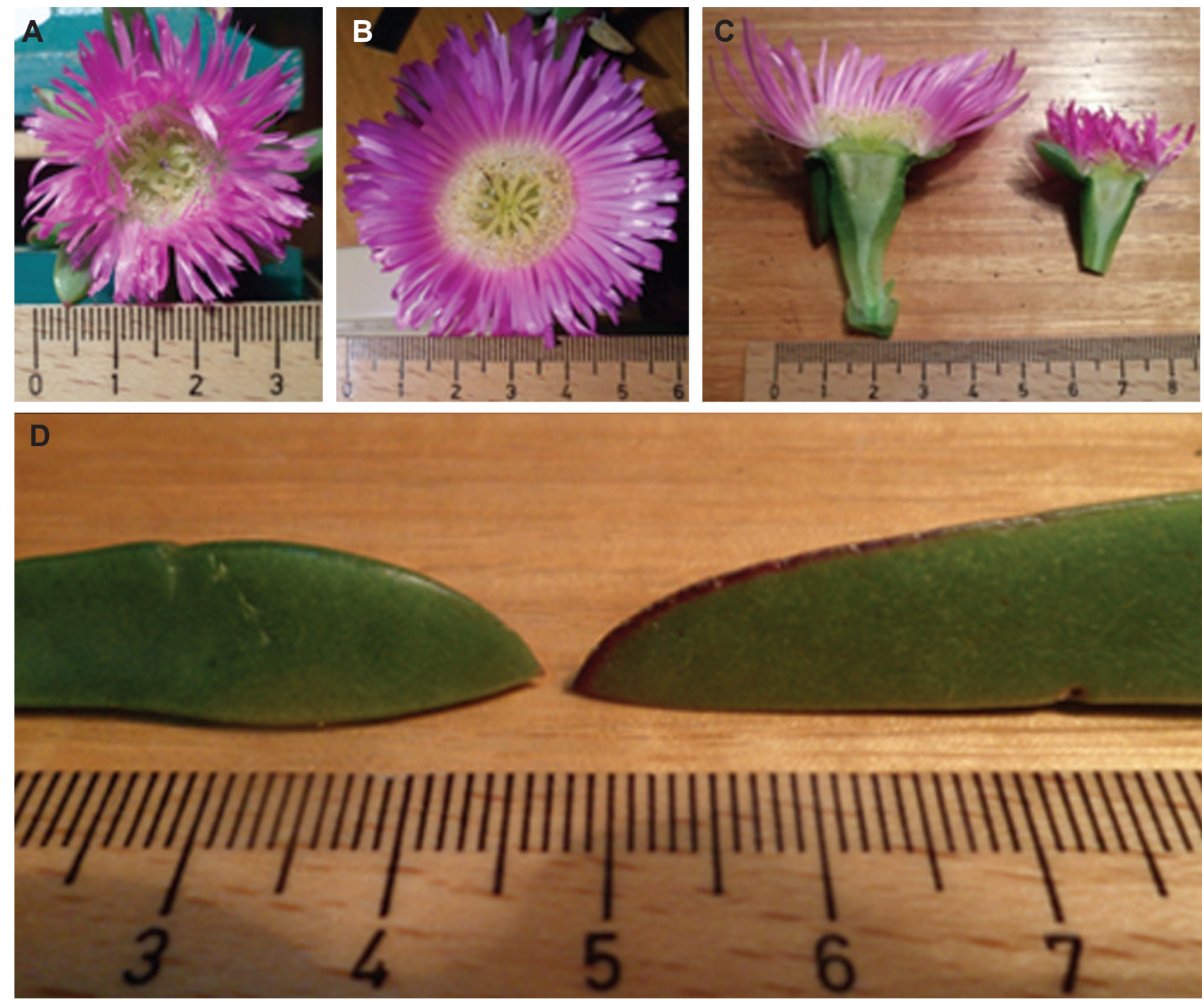

Figura 1. A. Flor de Carpobrotus chilensis. B. Flor de C. edulis. C. Corte longitudinal de flores de C. edulis (izquierda) y C. chilensis (derecha). D. Hojas de C. chilensis (izquierda) y C. edulis (derecha) con detalle de arista aserrada.

Figure 1. A. Flower of Carpobrotus chilensis. B. Flower of C. edulis. C. Longitudinal section of C. edulis (left) and C. chilensis (right) flowers. D. Leaves of C. chilensis (left) and C. edulis (right) with sawed edge detail.

y plumosos (Fig. 1 B y C). Época de floración primavera y verano. El fruto es una baya con cáliz persistente, indehiscente, carnoso y comestible de $2,5-3 \mathrm{~cm}$ de largo, se torna amarillo con el tiempo (DiTomaso \& Healy 2007). Semillas numerosas, ovoideas, algo comprimidas, inmersas en mucílago (Blake 1969, Castroviejo 1990). Los frutos son ampliamente dispersados por mamíferos y aves. Se reproduce vegetativamente y a través de semillas (DiTomaso \& Healy 2007).

De acuerdo a este nuevo registro, el género Carpobrotus queda representado en Chile por dos especies que se pueden separar por la siguiente clave:

1. Hojas de 3-5 cm de largo, no aserradas en la arista inferior; flores de 3-5 cm de diámetro; pétalos de color purpúreo (puro)...Carpobrotus chilensis 1'. Hojas de 7-10 cm de largo, aserradas en la arista inferior; flores de 6-10 cm de diámetro; pétalos de color amarillo, anaranjado o purpúreo con matices blancos.

Carpobrotus edulis 
Material estudiado

Región de Valparaíso: Provincia de Valparaíso: Quintero, Puntilla San Fuentes (32॰45'33"S-71'32'45"O), 16-X2013, G. Quiroz y R. Rodríguez 4782 (CONC No 177864); Viña del Mar, Las Salinas (32॰59'18"S-71³2'19'O), 15-VII-1931, F. Jaffuel s/n (CONC 46415). Provincia de

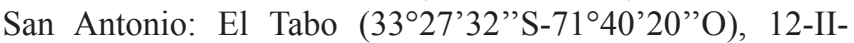
1952, H. Gunckel 23209 (CONC 109089); San Sebastián (3331'36"S-71³6'17'O), 3-I-1965. R. Acevedo (CONC 144750). Región del Maule: Provincia de Talca: Constitución (35'19'26'S-72²5'21'O), 14-XI-1959, H. Gunckel 35152 (CONC 109087). Región del Biobío: Provincia de Concepción: Cocholgüe en los acantilados (36 36'06”'S 72॰58'39”O), 10-X-2013, G. Sotes s/n (CONC N $\left.{ }^{\circ} 177863\right)$; Lenga (3646'41'S-73¹0'84”O), 25-IX-2013, G. Sotes \& R. Rodríguez 4781 (CONC N ${ }^{\circ}$ 177862); Tomé, sector Tres Pinos (36³8'60'S-7257'57'O), 23-XI-2013, G. Sotes s/n (CONC $\left.{ }^{\circ} 177865\right)$. Región de Los Ríos: Provincia de Valdivia: Mehuín (39²5’43”S-73¹2’54”O), 18-II-1976, G. Montero 10155 (CONC 107376).

\section{AGRADECIMIENTOS}

Este trabajo es parte de las actividades de investigación del Instituto de Ecología y Biodiversidad (IEB) financiado por FICM P05-002 y CONICYT PBF-023. También se agradece a la Comisión Nacional de Ciencia y Tecnología (CONICYT) por beca doctoral.

\section{BIBLIOGRAFÍA}

Albert, M.E., C.M. D’Antonio \& K.A. Schierenbeck. 1997. Hybridization and introgression in Carpobrotus spp. (Aizoaceae) in California. I. Morphological evidence. American Journal of Botany 84: 896-904.
Blake, S.T. 1969. A Revision of Carpobrotus and Sarcozoma in Australia, genera allied to Mesembryanthemum (Aizoaceae). Contribution from the Qeensland Herbarium 7: 1-65.

Castroviejo, S. 1990. Aizoaceae. En: S. Castroviejo, M. Laínz, G. López González, P. Montserrat, F. Muñoz Garmendia, J. Paiva \& L. Villar (eds.), Plantas vasculares de la Península Ibérica e Islas Baleares, Vol. 2, pp. 71-92.

DiTomaso, J.M. \& E.A Healy. 2007. Weeds of California and Other Western States. Univ. California, DANR. Publ. \#3488, 1808 pp.

GAY, C. 1848. Mesembriantemas. En: C. Gay (ed.), Historia Física y Política de Chile. Botánica, Vol. 3, pp. 5-7.

Global Invasive Species Database. URL: http://www.issg.org/ database/welcome/ Consultado: 3 diciembre, 2013.

Heenan, P.B. \& W.R. Sykes. 2010. Taxonomic notes on the New Zealand flora: Carpophyma mutabilis and Carpophyma pallida (Aizoaceae), new names for two wild intergeneric hybrids. New Zealand Journal of Botany 48: 225-230.

Muñoz Pizarro, C. 1966. Flores silvestres de Chile. Ediciones Universidad de Chile, Chile. 245 pp.

Munz, P.A. 1959. A California Flora. University of California Press, U.S.A. $1681 \mathrm{pp}$.

Novoa, A., L. González, L. Moravcová \& P. Pyšek. 2013. Constraints to native plant species establishment in coastal dune communities invaded by Carpobrotus edulis: Implications for restoration. Biological Conservation 164: $1-9$.

Novoa, A., R. Rodríguez, D. Richardson \& L. GonzÁlez. 2014. Soil quality: a key factor in understanding plant invasion? The case of Carpobrotus edulis (L.) N.E.Br. Biological Invasions 16: 429-443.

Reiche, C. 1898. Estudios críticos sobre la flora de Chile. Anales de la Universidad de Chile 100: 327-371.

Schmalzer, P. \& C. Hinkle. 1987. Species biology and potential for controlling four exotic plants (Ammophila arenaria, Carpobrotus edulis, Cortaderia jubata and Gasoul crystallinum on Vandenberg Air Force Base, California. The Bionetics Corporation, NASA, U.S.A. 103 pp.

Zuloaga, F., O. Morrone \& M. Belgrano (Eds.). 2008. Catálogo de las plantas vasculares del Cono Sur (Argentina, Sur de Brasil, Chile, Paraguay y Uruguay). Missouri Botanical Garden Press, U.S.A. 3348 pp. 